\title{
The effects of exercise and low-calorie diets compared with low-calorie diets alone on health: a protocol for systematic reviews and meta-analyses of controlled clinical trials
}

Sara Beigrezaei ${ }^{1,2}$, Zeinab Yazdanpanah ${ }^{1,2}$, Sepideh Soltani ${ }^{3}$, Seyede Hamide Rajaie ${ }^{1,2}$, Sahar Mohseni-Takalloo ${ }^{1,2,4}$, Tayebeh Zohrabi ${ }^{1,2}$, Mojtaba Kaviani ${ }^{5}$, Scott C. Forbes ${ }^{6}$, Julien S. Baker ${ }^{7}$ and Amin Salehi-Abargouei ${ }^{1,2^{*}}$ (D)

\begin{abstract}
Background: Exercise and weight loss diets are two independent non-pharmaceutical strategies used to improve several aspects of body composition and health. We plan to systematically review controlled clinical trials investigating weight loss diets alone compared to weight loss diets in conjunction with exercise on energy intake, body weight, body composition, cardiometabolic risk factors, sex hormones, and mental health.

Methods and analysis: PubMed/MEDLINE, EMBASE, ISI (Web of Science), Scopus, and Google Scholar will be searched to retrieve potential controlled clinical trials investigating the effects of exercise in conjunction with weight loss diets compared with weight loss diets alone on energy intake, body weight and composition (fat mass, fat-free mass), anthropometrics (waist circumference), cardiometabolic markers, sex hormones [testosterone, estradiol, and sex hormone binding globulin (SHBG)], liver and kidney enzymes (alanine aminotransferase (ALT), aspartate aminotransferase (AST), gamma-glutamyl transferase (GGT), uric acid, blood urea nitrogen (BUN), glomerular filtration rate (GFR), quality of life, and depression in adults. The weighted mean difference (WMD) and its corresponding 95\% confidence intervals (Cls) will be derived using random effects model. Several subgroup analyses based on follow-up duration, the health status of the participants, the diet used for weight loss, the exercise protocol, participants' sex, and other possible variables will be conducted to explore possible sources of heterogeneity. Publication bias will be explored by inspecting funnel plots and by conducting asymmetry tests. Overall quality of the evidence will be assessed by using the Grading of Recommendations Assessment, Development, and Evaluation (GRADE) tool.

(Continued on next page)
\end{abstract}

\footnotetext{
* Correspondence: abargouei@ssu.ac.ir; abargouei@gmail.com

'Nutrition and Food Security Research Center, Shahid Sadoughi University of Medical Sciences, Yazd, Iran

${ }^{2}$ Department of Nutrition, School of Public Health, Shahid Sadoughi University of Medical Sciences, Yazd, Iran

Full list of author information is available at the end of the article
}

(c) The Author(s). 2021 Open Access This article is licensed under a Creative Commons Attribution 4.0 International License, which permits use, sharing, adaptation, distribution and reproduction in any medium or format, as long as you give appropriate credit to the original author(s) and the source, provide a link to the Creative Commons licence, and indicate if changes were made. The images or other third party material in this article are included in the article's Creative Commons. licence, unless indicated otherwise in a credit line to the material. If material is not included in the article's Creative Commons licence and your intended use is not permitted by statutory regulation or exceeds the permitted use, you will need to obtain permission directly from the copyright holder. To view a copy of this licence, visit http://creativecommons.org/licenses/by/4.0/. The Creative Commons Public Domain Dedication waiver (http://creativecommons.org/publicdomain/zero/1.0/) applies to the data made available in this article, unless otherwise stated in a credit line to the data. 
(Continued from previous page)

Discussion: We envisage that this systematic review and meta-analysis will provide valuable information regarding the effectiveness of adding exercise to weight loss diets. No primary data is going to be collected; therefore, ethical approval is not required. The resulting manuscripts will be disseminated in peer-reviewed journals and at international and national conferences.

Systematic review registration: The study protocol is registered in the International Prospective Register of Systematic Reviews (PROSPERO, Registration ID: CRD42020173434).

Keywords: Diet, Diet plus exercise, Energy intake, Cardiometabolic markers, Mental health, Bone health, Systematic review, Meta-analysis

\section{Background}

The worldwide prevalence of obesity and associated metabolic abnormalities has resulted in a huge strain on health care systems $[1,2]$. The increase in prevalence of obesity in recent decades is multifactorial; however, sedentary lifestyle and poor quality diets are proposed to be the two major contributing factors [2, 3]. Furthermore, obesity is associated with a reduced quality of life and higher risk factors for several diseases, such as metabolic syndrome, diabetes, cardiovascular disease (CVD), and cancers $[4,5]$.

Lifestyle modifications including changes in diet and physical activity are regarded as the main non-pharmacological and non-surgical strategies to treat obesity [6]. Modified dietary macro-nutrient intake leading to a hypocaloric diet is effective for weight loss over the short term and may be important for weight loss maintenance compared to exercise alone. Low-calorie diets not only reduce body weight but also improve cardiometabolic health, quality of life, and mental health [7-14]; however, it is proposed that weight loss diets might adversely affect bone health in adults [15]. It is also plausible that exercise may alter energy balance and influence body weight and health over time. Despite the well-known benefits of exercise, the increase in energy expenditure and the potential to decrease hunger and energy intake exercise alone does not seem to be effective at modifying weight status [16-18]. Beyond weight loss, exercise, may modulate metabolism and lead to an increase in muscle mass [18-21]. Furthermore, exercise (particularly weight-bearing exercise) may be effective at enhancing bone health [15].

In theory, exercise in conjunction with weight loss diets may be ideal to improve weight loss as well as appetite (i.e., energy intake), body composition, cardiovascular health and mental health [22-24]. However, controlled clinical trials have led to inconsistent results [25-29], with some studies demonstrating no additive effects of exercise $[25,26]$, while others found a greater effect with exercise for improving multiple cardiometabolic risk factors in obese adults $[27,28]$. A number of clinical trials have revealed that subjects show a significant weight loss and reduction in energy intake during an exercise intervention, while others have shown less reduction in body weight due to an increase in energy intake [30-32]. These conflicting results were also observed on other health outcomes such as bone health, appetite, and mood [33-37].

A number of systematic reviews and meta-analyses have compared the effects of diet, exercise or both in specific health conditions $[38,39]$. Hemmingsen et al. [38] reported no differences between the effects of diet alone or exercise alone compared to a standard treatment on the risk of type 2 diabetes mellitus and related complications. In addition, another systematic review evaluated the effects of diet or exercise or both on excessive weight gain during pregnancy and showed that diet or exercise alone and diet plus exercise during pregnancy appears to reduce the risk of excessive gestational weight gain [39]. Aside from the aforementioned reviews, we are not aware of any systematic review attempting to summarize the current evidence on other outcomes such as bone health, sex hormones, liver and kidney enzymes, quality of life, and depression.

\section{Objectives}

In this study, we will describe the protocols used to systematically compare the effects of a low-calorie diet with a low-calorie diet plus exercise on energy intake, body weight and composition, anthropometric measures, cardiometabolic markers, bone health markers, sex hormones, liver and kidney enzymes, quality of life, and depression in adults.

\section{Methods}

The present protocol is being reported in accordance with the reporting guidance provided in the Preferred Reporting Items for Systematic Reviews and MetaAnalyses Protocols (PRISMA-P) statement [40] (see checklist in Additional file 1). The study protocol is also registered in the International Prospective Register of Systematic Reviews (PROSPERO, Registration ID: CRD42020173434).

\section{Information sources and search strategy}

The relevant articles will be identified in the following databases up to August 2020: PubMed/MEDLINE, 
EMBASE, ISI (Web of Science), Scopus, and Google Scholar using Medical Subject Heading (MeSH) and non-MeSH keywords. We will not apply any language or other restrictions. In addition, we will check the reference lists of all relevant studies to identify additional relevant articles. Unpublished studies will be identified by searching the websites indexing the preprints such as Research Square (https://www.researchsquare.com/) and the registered clinical trials approved by the World Health Organization (WHO). All abstracts of interest will be evaluated for further information by contacting the authors. The draft searches for the main databases are available in Additional file 2.

\section{Study selection}

Two investigators will independently perform the study selection. All articles from electronic searches will be imported into the EndNote software (version: desktop, X7; Thompson Reuters, New York, USA) and duplicate studies will be deleted. Titles, abstracts, and full-text articles will be screened and cross-checked according to the eligibility criteria for study inclusion independently by 6 reviewers (Z.Y, S.S, S.B, SH.R, S.MT, and T.Z). Any disagreements will be resolved by discussion and consensus. The PRISMA flow chart will be presented to describe the process of the study selection.

Articles selected for full-text review must meet the following criteria:

(i) Participants must be a minimum 18 years of age and older and have a body mass index (BMI) $\geq 25$ $\mathrm{kg} / \mathrm{m}^{2}$ (pregnant and lactating women will be excluded);

(ii) Interventions must contain one arm in which participants receive an exercise intervention (i.e., aerobic or resistance) with a weight loss (i.e., hypocaloric) diet and one arm where participants only receive a weight loss diet (exactly according to the diet considered for the intervention group);

(iii) Interventions must be randomized or nonrandomized controlled clinical trials with either a parallel, cross-over, or factorial design with at least 2 weeks of follow-up.

\section{Data extraction and management}

The following data will be extracted using a predefined data extraction form by two independent investigators from the eligible studies and any discrepancy will be resolved by a third author:

\section{Study and participant's characteristics}

The participants' age, number of males and females, number of participants in the intervention and control group/period, the geographical location of the study, and the health condition of participants.

\section{Intervention details}

The study design (parallel/cross-over/factorial), number of study arms, the intervention duration, funding source(s), amount of calorie restriction, type of diets and exercise programs, intensity, frequency, compliance, and delivery of each exercise used for the intervention group.

\section{Outcome measures}

Data on baseline, post-intervention, or change from baseline mean \pm standard deviation (SD) for energy intake, body weight, anthropometrics and body composition measures, blood glucose control markers (serum/ plasma fasting glucose, insulin, insulin resistance markers including HOMA-IR and hemoglobin A1C), lipid profile [serum total cholesterol, triglycerides, lowdensity lipoprotein cholesterol (LDL-C), high-density lipoprotein cholesterol (HDL-C), and apoproteins], systolic and diastolic blood pressure, sexual hormones (testosterone and estradiol), SHBG, serum/plasma inflammation (hs-CRP, IL-6, and TNF-a), bone health markers, liver and kidney enzymes, depression, and quality of life, will be extracted for the intervention and control groups/periods. $P$ values for within-group and between-group comparison will also be collected to calculate the change values.

\section{Assessment of risk of bias in individual studies}

The eligible randomized trials will be assessed using the Cochrane collaboration's risk of bias assessment tool considering seven domains: (i) random sequence generation (selection bias), (ii) allocation concealment (selection bias), (iii) blinding of participants and personnel (performance bias), (iv) blinding of outcome assessment (detection bias), (v) incomplete outcome data (attrition bias), (vi) selective reporting (reporting bias), and (vii) the dietary compliance as another possible source of bias in dietary interventions. Each study will be judged as low risk of bias, high risk of bias, or unclear risk of bias according to the mentioned domains [41]. The overall quality of studies will be classified as low risk (low risk for all domains), unclear risk (unclear for at least one domain), and high risk (high risk for at least one domain). As well, the non-randomized trials will be evaluated using risk of bias in non-randomized studies of interventions (ROBINS-I) tool [42]. According this tool, bias will be examined based on 7 domains (i.e., confounding factors, selection of participants, interventions classification, deviations from intended interventions, missing data, outcomes measurement, and selective reporting), and then reporting an overall risk of bias (i.e., low, moderate, serious, critical, or no information). 


\section{Data analysis}

The data for study characteristics, participants, outcomes, and findings will be used to build evidence tables for eligible studies to provide an overall description of included studies. The mean change values from baseline for the intervention (weight loss diet + exercise) and control group/period (weight loss diet alone) and their standard deviations (SDs) will be used to calculate the raw mean difference and standard error (SE) between the intervention and control. The hedges' $g$ (bias corrected standardized mean difference) statistic and corresponding SD will be calculated for outcome variables reported in different scales. The mean difference will be used as the effect size for meta-analysis. If the change values were not reported, we will calculate $\mathrm{SD}$ for the change values by selecting 0.5 as the reference correlation coefficient between baseline and end point values $(r=0.5)$ and to make sure that the meta-analysis was not sensitive to the selected correlation coefficient, all analyses will be repeated using 0.2 and 0.8 as the correlation coefficient. The weighted mean difference (WMD) and its corresponding 95\% confidence intervals (CIs) will be derived using the random effects model which takes the between-study heterogeneity into account [43]. All statistical analyses will be performed using STATA, version 11.2 (Stata Corp, College Station, TX) and a twosided $P$ value less than 0.05 will be considered as statistically significant. If data cannot be meta-analyzed, we will summarize the articles and conclude on high-quality studies.

\section{Between study heterogeneity and subgroup analysis}

The heterogeneity will be checked using Cochran's $Q$ test and $I^{2}$ statistic ( $I^{2}$ is an estimate for between study variation to total meta-analysis variation ratio ranging from 0 to $100 \%$ ) [44]. We will report Cochran $Q$ test with a $P$ value of $<0.05$ considered statistically significant (heterogeneity). $I^{2}$ with values of $0-25 \%$ and $75-$ $100 \%$ will be taken to indicate low and considerable heterogeneity, respectively. To examine the potential sources of between-study heterogeneity, several subgroup analyses based on follow-up duration, the health status of the participants, the diet used for weight loss, the exercise, participants' sex, and other possible variables will be conducted.

\section{Sensitivity analysis}

The sensitivity analysis will be done by sequentially removing individual studies included in the meta-analyses to assess the robustness of the meta-analyses [45].

\section{Publication bias}

In case there are fewer than 10 studies in a metaanalysis, we will construct a funnel plot to investigate the potential for publication bias for the primary outcome by visual inspection for asymmetry. If our metaanalysis involves 10 or more studies, publication bias will be evaluated by inspecting Begg's funnel plots and Egger's and Begg's asymmetry tests [46]. Duval and Tweedie's trim and fill analysis will be conducted if the publication bias becomes evident [47].

\section{Dealing with missing data}

If data are missing, we will attempt to contact the authors through e-mails to obtain missing data or additional information twice, 1 week apart. The impact of missing data will also be evaluated in the sensitivity analysis. Additionally, we will describe the possible influences of missing data in the "Discussion" section of the resulting publications.

\section{Confidence in cumulative evidence}

Overall quality of the evidence will be assessed by using the Grading of Recommendations Assessment, Development and Evaluation (GRADE) tool [48] with GRAD Eprofiler (GRADEpro) V.3.6 software, identifying the quality of evidence for each outcome as the extent to which one can be confident that an estimate of effect is near to the quantity of certain interest [46]. There are four levels used to rate the quality of evidence across trials in the GRADE system: very low, low, moderate, and high. Randomized clinical trials are categorized as high quality but can be downgraded due to limitation in study design, indirectness of evidence, imprecision of results, unexplained heterogeneity or inconsistency of results, or high probability of publication bias [48].

\section{Discussion}

For decades, epidemiological and clinical studies have been elucidating the link between lifestyle modifications and health outcomes through different mechanisms [49]. Previous reviews have assessed the impacts of diet or exercise alone on energy intake and different health indicators, while there is no comprehensive investigation summarizing the evidence evaluating the effects of weight loss diets combined with exercise interventions on energy intake, anthropometric and body composition, blood glucose control, cardio-metabolic markers, and mental health. Nevertheless, this systematic review and meta-analysis might face several potential limitations. High heterogeneity between included studies might arise from differences in study characteristics not anticipated by authors or not explained at study level. The limited number of studies particularly RCTs with risk of bias on some outcome variables might lead to inconclusive results.

In this manuscript, we present the study protocol for a systematic review and meta-analysis to compare the 
effects of a low-calorie diet plus exercise with a lowcalorie diet on risk factors associated with chronic diseases. Finally, this systematic review and meta-analyses will provide more information regarding the effectiveness of adding exercise to a weight loss diet.

\begin{abstract}
Abbreviations
ALT: Alanine aminotransferase; AST: Aspartate aminotransferase; BMI: Body mass index; BUN: Uric acid, blood urea nitrogen; Cl: Confidence interval; CVD: Cardiovascular disease; GFR: Glomerular filtration rate; GGT: Gammaglutamyl transferase; HDL-C: High-density lipoprotein cholesterol; hsCRP: Highly sensitive C-reactive protein; IL-6: Interleukin 6; LDL-C: Lowdensity lipoprotein cholesterol; MeSH: Medical Subject Headings; PRISMAP: Preferred Reporting Items for Systematic Reviews and Meta-Analyses Protocols; PROSPERO: International Prospective Register of Systematic Reviews; SD: Standard deviation; SE: Standard error; SHBG: Sex hormone-binding globulin; TNF-a: Tumor necrosis factor alpha; WHO: World Health Organization; WMD: Weighted mean difference
\end{abstract}

\section{Supplementary Information}

The online version contains supplementary material available at https://doi. org/10.1186/s13643-021-01669-7.

Additional file 1. PRISMA-P 2015 Checklist.

Additional file 2. Search strategies used to find related publications in PubMed, Scopus and ISI web of science.

\section{Acknowledgements}

We would like to thank the research council of Nutrition and Food Security Research Center for their scientific support.

\section{Authors' contributions}

ASA conceived the study and will be the guarantor of the review. ASA, ZY, and SS contributed in defining the search strategy. SB wrote the first draft of the manuscript. ZY, SS, SHR, SMT, TZ, MK, and SF facilitated with preparation of the manuscript and its finalization. All authors read and approved the final version of the manuscript.

\section{Funding}

The present systematic review was supported by the Research Council of the Nutrition and Food Security Research Center, Shahid Sadoughi University of Medical Sciences, Yazd, Iran.

\section{Availability of data and materials}

The studies included in the review will be available upon request.

\section{Declarations}

\section{Ethics approval and consent to participate}

We will follow the Preferred Reporting Items for Systematic Reviews and Meta-analysis (PRISMA) for reporting the present systematic review and meta-analysis. The protocol is undergoing registration in the International Prospective Register of Systematic Reviews (PROSPERO) database.

\section{Consent for publication}

No individual detail is presented in this protocol; therefore, it is not applicable.

\section{Competing interests}

The authors declare that they have no competing interests.

\section{Author details}

'Nutrition and Food Security Research Center, Shahid Sadoughi University of Medical Sciences, Yazd, Iran. 'Department of Nutrition, School of Public Health, Shahid Sadoughi University of Medical Sciences, Yazd, Iran. ${ }^{3}$ Yazd Cardiovascular Research Center, Shahid Sadoughi University of Medical Sciences, Yazd, Iran. ${ }^{4}$ School of Medicine, Bam University of Medical Sciences, Bam, Iran. ${ }^{5}$ Faculty of Pure \& Applied Science, School of Nutrition and
Dietetics, Acadia University, Wolfville, NS, Canada. ${ }^{6}$ Department of Physical Education Studies, Faculty of Education, Brandon University, Brandon, MB, Canada. ${ }^{7}$ Centre for Health and Exercise Science Research, Head, Department of Sport, and Physical Education, Hong Kong Baptist University, Kowloon Tong, Hong Kong.

Received: 23 May 2020 Accepted: 7 April 2021

Published online: 20 April 2021

\section{References}

1. Brewer CJ, Balen AH. Focus on obesity. Reproduction. 2010;140(3):347-64. https://doi.org/10.1530/REP-09-0568.

2. Finkelstein EA, Khavjou OA, Thompson H, Trogdon JG, Pan L, Sherry B, et al. Obesity and severe obesity forecasts through 2030. American journal of preventive medicine. 2012;42(6):563-70. https://doi.org/10.1016/j.amepre.2 011.10.026.

3. World Health Organization. Obesity and overweight, Available at https:// www.who.int/news-room/fact-sheets/detail/obesity-and-overweight. Access in 16 February 2018

4. Fox CS, Golden SH, Anderson C, Bray GA, Burke LE, De Boer IH, et al. Update on prevention of cardiovascular disease in adults with type 2 diabetes mellitus in light of recent evidence: a scientific statement from the American Heart Association and the American Diabetes Association. Circulation. 2015:132(8):691-718. https://doi.org/10.1161/CIR. 0000000000000230

5. Després J-P. Body fat distribution and risk of cardiovascular disease: an update. Circulation. 2012;126(10):1301-13. https://doi.org/10.1161/CIRCULA TIONAHA.111.067264.

6. Wadden TA, Webb VL, Moran CH, Bailer BA. Lifestyle modification for obesity: new developments in diet, physical activity, and behavior therapy. Circulation. 2012;125(9):1157-70. https://doi.org/10.1161/CIRCULATIONA HA.111.039453.

7. Miller GD, Beavers D, Hamm D, Mihalko S, Messier S. Nutrient intake during diet-induced weight loss and exercise interventions in a randomized trial in older overweight and obese adults. The journal of nutrition, health \& aging. 2017;21(10):1216-24. https://doi.org/10.1007/ s12603-017-0892-5.

8. Messier SP, Mihalko SL, Legault C, Miller GD, Nicklas BJ, DeVita P, et al. Effects of intensive diet and exercise on knee joint loads, inflammation, and clinical outcomes among overweight and obese adults with knee osteoarthritis: the IDEA randomized clinical trial. Jama. 2013:310(12):1263-73. https://doi.org/10.1001/jama.2013.277669.

9. Aaboe J, Bliddal H, Messier S, Alkjaer T, Henriksen M. Effects of an intensive weight loss program on knee joint loading in obese adults with knee osteoarthritis. Osteoarthritis and Cartilage. 2011;19(7):822-8. https://doi.org/1 0.1016/j.joca.2011.03.006

10. Grandy S, Hashemi M, Langkilde AM, Parikh S, Sjöström CD. Changes in weight loss-related quality of life among type 2 diabetes mellitus patients treated with dapagliflozin. Diabetes, Obesity and Metabolism. 2014;16(7): 645-50. https://doi.org/10.1111/dom.12263.

11. Singh RB, Watanabe S, Li D, Nakamura T, Juneja LR, Takahashi T, et al. Diet and lifestyle guidelines and desirable levels of risk factors and protective factors for prevention of dementia-a scientific statement from Joint Symposium Of Jaas And Apcns. Biomedical Journal of Scientific \& Technical Research. 2019;17(3):12844-64.

12. Blaine BE, Rodman J, Newman JM. Weight loss treatment and psychological well-being: a review and meta-analysis. J Health Psychol. 2007;12(1):66-82. https://doi.org/10.1177/1359105307071741.

13. Wahlroos S, Phillips ML, Lewis MC, Kow L, Toouli J, Slavotinek JP, et al. Rapid significant weight loss and regional lipid deposition: implications for insulin sensitivity. Obesity research \& clinical practice. 2007;1 (1):1-78. https://doi. org/10.1016/j.orcp.2006.08.002

14. Ashtary-Larky D, Ghanavati M, Lamuchi-Deli N, Payami SA, Alavi-Rad S, Boustaninejad M, et al. Rapid weight loss vs. slow weight loss: which is more effective on body composition and metabolic risk factors? Int J Endocrinol Metab. 2017;15(3):e13249.

15. Soltani S, Hunter GR, Kazemi A, Shab-Bidar S. The effects of weight loss approaches on bone mineral density in adults: a systematic review and meta-analysis of randomized controlled trials. Osteoporos Int. 2016;27(9): 2655-71. https://doi.org/10.1007/s00198-016-3617-4. 
16. Garrow JS, Summerbell CD. Meta-analysis: effect of exercise, with or without dieting, on the body composition of overweight subjects. Eur J Clin Nutr. 1995;49(1):1-10.

17. Pontzer H, Durazo-Arvizu R, Dugas LR, Plange-Rhule J, Bovet P, Forrester $T E$, et al. Constrained total energy expenditure and metabolic adaptation to physical activity in adult humans. Curr Biol. 2016;26(3):410-7. https://doi. org/10.1016/j.cub.2015.12.046.

18. Martin M, Krystof S, Martina D, Renata V, Ondrej M, Stepan S, et al. Modulation of energy intake and expenditure due to habitual physical exercise. Current pharmaceutical design. 2016;22(24):3681-99. https://doi. org/10.2174/1381612822666160419144200.

19. Thompson D, Karpe F, Lafontan M, Frayn K. Physical activity and exercise in the regulation of human adipose tissue physiology. Physiological reviews. 2012;92(1):157-91. https://doi.org/10.1152/physrev.00012.2011.

20. Arent SM, Walker AJ, Pellegrino JK, Sanders DJ, McFadden BA, Ziegenfuss $\mathrm{TN}$, et al. The combined effects of exercise, diet, and a multi-ingredient dietary supplement on body composition and adipokine changes in overweight adults. Journal of the American College of Nutrition. 2018;37(2): 111-20. https://doi.org/10.1080/07315724.2017.1368039.

21. Arner P, Andersson DP, Bäckdahl J, Dahlman I, Rydén M. Weight gain and impaired glucose metabolism in women are predicted by inefficient subcutaneous fat cell lipolysis. Cell Metab. 2018;28(1):45-54 e3.

22. Abbenhardt C, McTiernan A, Alfano CM, Wener MH, Campbell KL, Duggan $C$, et al. Effects of individual and combined dietary weight loss and exercise interventions in postmenopausal women on adiponectin and leptin levels. Journal of internal medicine. 2013;274(2):163-75. https://doi.org/10.1111/ joim.12062.

23. Imayama I, Alfano CM, Kong A, Foster-Schubert KE, Bain CE, Xiao L, et al. Dietary weight loss and exercise interventions effects on quality of life in overweight/obese postmenopausal women: a randomized controlled trial. International Journal of Behavioral Nutrition and Physical Activity. 2011;8(1): 118. https://doi.org/10.1186/1479-5868-8-118.

24. Weiss EP, Albert SG, Reeds DN, Kress KS, McDaniel JL, Klein S, et al. Effects of matched weight loss from calorie restriction, exercise, or both on cardiovascular disease risk factors: a randomized intervention trial. The American journal of clinical nutrition. 2016;104(3):576-86. https://doi.org/1 0.3945/ajen.116.131391.

25. Christiansen T, Paulsen SK, Bruun JM, Pedersen SB, Richelsen B. Exercise training versus diet-induced weight-loss on metabolic risk factors and inflammatory markers in obese subjects: a 12-week randomized intervention study. American Journal of Physiology-Endocrinology and Metabolism. 2010; 298(4):E824-E31. https://doi.org/10.1152/ajpendo.00574.2009.

26. Fayh APT, Lopes AL, Fernandes PR, Reischak-Oliveira A, Friedman R. Impact of weight loss with or without exercise on abdominal fat and insulin resistance in obese individuals: a randomised clinical trial. British journal of nutrition. 2013;110(3):486-92. https://doi.org/10.1017/S0007114512005442.

27. García-Unciti M, Izquierdo M, Idoate F, Gorostiaga E, Grijalba A, OrtegaDelgado F, et al. Weight-loss diet alone or combined with progressive resistance training induces changes in association between the cardiometabolic risk profile and abdominal fat depots. Annals of Nutrition and Metabolism. 2012;61(4):296-304. https://doi.org/10.1159/000342467.

28. Ibáñez J, Izquierdo M, Martínez-Labari C, Ortega F, Grijalba A, Forga L, et al. Resistance training improves cardiovascular risk factors in obese women despite a significative decrease in serum adiponectin levels. Obesity. 2010; 18(3):535-41. https://doi.org/10.1038/oby.2009.277.

29. Svendsen O, Hassager C, Christiansen C. Six months' follow-up on exercise added to a short-term diet in overweight postmenopausal women--effects on body composition, resting metabolic rate, cardiovascular risk factors and bone. International journal of obesity and related metabolic disorders: journal of the International Association for the Study of Obesity. 1994;18(10): 692-8.

30. King NA, Hopkins M, Caudwell P, Stubbs R, Blundell JE. Individual variability following 12 weeks of supervised exercise: identification and characterization of compensation for exercise-induced weight loss. International journal of obesity. 2008;32(1):177-84. https://doi.org/10.1038/sj. ijo.0803712.

31. Schubert MM, Desbrow B, Sabapathy S, Leveritt M. Acute exercise and subsequent energy intake. A meta-analysis. Appetite. 2013;63:92-104. https://doi.org/10.1016/j.appet.2012.12.010.

32. King JA, Miyashita M, Wasse LK, Stensel DJ. Influence of prolonged treadmill running on appetite, energy intake and circulating concentrations of acylated ghrelin. Appetite. 2010;54(3):492-8. https://doi.org/10.1016/j.appet.2 010.02.002.

33. Andersen RE, Wadden TA, Herzog RJ. Changes in bone mineral content in obese dieting women. Metabolism. 1997;46(8):857-61. https://doi.org/10.101 6/S0026-0495(97)90070-6.

34. Anton S, Han H, York E, Martin C, Ravussin E, Williamson D. Effect of calorie restriction on subjective ratings of appetite. Journal of human nutrition and dietetics. 2009;22(2):141-7. https://doi.org/10.1111/j.1365-277X.2008.00943.X.

35. De Jonge L, Moreira EA, Martin CK, Ravussin E, Team PC. Impact of 6-month caloric restriction on autonomic nervous system activity in healthy, overweight, individuals. Obesity. 2010;18(2):414-6. https://doi.org/10.1038/ oby.2009.408.

36. Yadav R, Yadav RK, Khadgawat R, Pandey RM. Comparative efficacy of a 12 week yoga-based lifestyle intervention and dietary intervention on adipokines, inflammation, and oxidative stress in adults with metabolic syndrome: a randomized controlled trial. Transl Behav Med. 2019;9(4):594604. https://doi.org/10.1093/tbm/iby060.

37. Redman LM, Rood J, Anton SD, Champagne C, Smith SR, Ravussin E. Calorie restriction and bone health in young, overweight individuals. Archives of internal medicine. 2008;168(17):1859-66. https://doi.org/10.1001/archinte.1 68.17.1859.

38. Hemmingsen B, Gimenez-Perez G, Mauricio D, Roqué I Figuls M, Metzendorf MI, Richter B. Diet, physical activity or both for prevention or delay of type 2 diabetes mellitus and its associated complications in people at increased risk of developing type 2 diabetes mellitus. Cochrane Database Syst Rev. 2017;12(12):CD003054. https://doi.org/10.1002/14651858.CD003054.pub4.

39. Muktabhant B, Lawrie TA, Lumbiganon P, Laopaiboon M. Diet or exercise, or both, for preventing excessive weight gain in pregnancy. Cochrane database of systematic reviews. 2015;6.

40. Moher D, Shamseer L, Clarke M, Ghersi D, Liberati A, Petticrew M, et al. Preferred reporting items for systematic review and meta-analysis protocols (PRISMA-P) 2015 statement. Systematic reviews. 2015;4(1):1. https://doi.org/1 0.1186/2046-4053-4-1.

41. Higgins JPT, Green S. Cochrane Handbook for Systematic Reviews of Interventions Version 5.1.0 [updatedMarch 2011]: The Cochrane Collaboration. 2021. Available from https://handbook-5-1.cochrane.org/. Accessed 18 Apr 2021

42. Sterne JA, Hernán MA, Reeves BC, Savović J, Berkman ND, Viswanathan M, Henry D, Altman DG, Ansari MT, Boutron I, Carpenter JR, Chan AW, Churchill R, Deeks JJ, Hróbjartsson A, Kirkham J, Jüni P, Loke YK, Pigott TD, Ramsay $C R$, Regidor D, Rothstein HR, Sandhu L, Santaguida PL, Schünemann HJ, Shea B, Shrier I, Tugwell P, Turner L, Valentine JC, Waddington H, Waters E, Wells GA, Whiting PF, Higgins JP. ROBINS-I: a tool for assessing risk of bias in non-randomised studies of interventions. BMJ. 2016;355:14919. https://doi. org/10.1136/bmj.i4919.

43. DerSimonian R, Laird N. Meta-analysis in clinical trials. Control Clin Trials. 1986;7(3):177-88. https://doi.org/10.1016/0197-2456(86)90046-2.

44. Higgins JP, Thompson SG. Quantifying heterogeneity in a meta-analysis. Stat Med. 2002;21(11):1539-58. https://doi.org/10.1002/sim.1186.

45. Egger M, Davey-Smith G, Altman D. Systematic reviews in health care: metaanalysis in context: John Wiley \& Sons; 2008.

46. Higgins JP, Green S. Cochrane handbook for systematic reviews of interventions: John Wiley \& Sons; 2011

47. Duval S, Tweedie R. Trim and fill: A simple funnel-plot-based method of testing and adjusting for publication bias in meta-analysis. Biometrics. 2000; 56(2):455-63. https://doi.org/10.1111/j.0006-341X.2000.00455.x.

48. Guyatt GH, Oxman AD, Vist GE, Kunz R, Falck-Ytter Y, Alonso-Coello P, et al. GRADE: an emerging consensus on rating quality of evidence and strength of recommendations. Bmj. 2008;336(7650):924-6. https://doi.org/10.1136/ bmj.39489.470347.AD.

49. Joumard I, André C, Nicq C, Chatal O. Health status determinants: lifestyle, environment, health care resources and efficiency. Environment, Health Care Resources and Efficiency (May 27, 2010) OECD Economics Department Working Paper. 2010(627).

\section{Publisher's Note}

Springer Nature remains neutral with regard to jurisdictional claims in published maps and institutional affiliations. 